\title{
Analysis of the ambiguity of log-constrained seismic impedance inversion
}

\author{
Li Guofa ${ }^{1,2}$, Li Hao ${ }^{1,2}$, Ma Yanyan ${ }^{1,2}$ and Xiong Jinliang ${ }^{3}$ \\ ${ }^{1}$ State Key Laboratory of Petroleum Resources and Prospecting, China University of Petroleum (Beijing), Beijing 102249, \\ China \\ ${ }^{2}$ Key Laboratory of Geophysical Prospecting, China National Petroleum Corporation, Beijing 102249, China \\ ${ }^{3}$ Dagang Oil field, PetroChina, Tianjin 300280, China
}

(C) China University of Petroleum (Beijing) and Springer-Verlag Berlin Heidelberg 2011

\begin{abstract}
Although the ambiguity of seismic inversion is widely recognized in both theory and practice, so far as a concrete inversion example is concerned, there is not any objective, controllable method or any standard for how to evaluate and determine its ambiguity and reliability, especially for the high frequency components beyond the effective seismic frequency band. Taking log-constrained impedance inversion as an example, a new appraisal method is proposed on the basis of analyzing a simple geological model. Firstly, the inverted impedance model is transformed to a reflection coefficient series. Secondly, the maximum effective frequency of the real seismic data is chosen as a cutoff point and the reflection coefficient series is decomposed into two components by low-pass and high-pass filters. Thirdly, the geometrical reflection characteristics of the high-frequency components and that of the real seismic data are compared and analyzed. Then, the reliability of the inverted impedance model is appraised according to the similarity of geometrical characteristics between the high-frequency components and the real seismic data. The new method avoids some subjectivity in appraising the inverted result, and helps to enhance the reliability of reservoir prediction by impedance inversion technology.
\end{abstract}

Key words: Log-constrained, impedance, reflection coefficients, seismic inversion, ambiguity

\section{Introduction}

The research into seismic inversion is one of the most active topics in seismic exploration. Seismic inversion in practice has been extended from exploration to exploitation, and even to time-lapse seismic and reservoir monitoring. As one method, it has been developed from acoustic to elastic impedance inversion (Connolly, 1999; Yang et al, 2007) and from linear to nonlinear inversion (Puryear et al, 2008; Velis, 2008; Huang et al, 2009). Although the ambiguity of seismic inversion is widely recognized both by geophysicists and geologists, standards for objectivity and operability, which can be used to evaluate and determine the ambiguity and reliability, are still absent. The ambiguity, which has held up practical research work in exploration and exploitation more and more seriously, gives rise to questions and arguments about its capability of solving practical geological problems.

Li (1998) analyzed the ambiguity of band limited wavelet inversion in detail and gave an inversion strategy. That is, the medium-frequency components of impedance are derived from seismic inversion and the low and high frequency components of impedance are from interpolation of logging

*Corresponding author. email: lgfseismic@126.com Received May 20, 2010 data. The three components are added to get the final seismic inversion result. Ma (2000) studied the effect of the initial impedance model on the inversion result and concluded that even with the constraint of logging and geological data, the ambiguity is not yet avoided. Yao (2000) analyzed the model-based inversion method, and realized that ambiguity is an intrinsic characteristic of seismic inversion which mainly depends on consistency between the initial model and subsurface geology, and that it is inadvisable to demand too high a resolution or to put more emphasis on the consistency between inversion impedance and logging data around the well. Chen (2003) discussed the misunderstanding of seismic inversion, and emphasized that "impedance inversion is an effective way of reservoir prediction. However, geologists cannot rely on it too much". Zhang (2007) analyzed statistically the inversion results according to tens of logging data sets in the Jiyang depression, and found that "the constraint on high-frequency components must be weakened to ensure the validity of inversion data". Fu (2004) and $\mathrm{Wu}$ et al (2008) concluded from the view of information theory that most of the geophysical process are irreversible and the results of reservoir prediction are not exclusive. Ancis (1997), Juve (1997), Ghosh (2000) and Cerney (2007) analyzed and discussed the ambiguity and resulting interpretation traps from different perspectives. 
With the ambiguity of log-constrained impedance inversion recognized, it is a matter of course that geologists are much more concerned with the appraisal and quality control of inversion results. Latimer (2000) discussed and analyzed the problems in the inversion process and gave a basic concept from a geologist's viewpoint to control and evaluate the inversion result according to the consistency of inversion results with logging data and the agreement of the synthetic seismogram with the real seismic record. Fu (1995) and Cui et al (2007) described the self-affine similarity between different frequency components of seismic data, Qin (2009) evaluated the reliability of inversion results by using similarity. In practice, geologists usually evaluate and determine the inversion result without well data ('blind-well'). In the meantime, they emphasize geological implications of inversion results. Although the appraisal method has been criticized and its scientific basis has been questioned by geophysicists, due to the lack of a new practicable appraisal method, the evaluation of inversion is still dependent mainly on the interpreters' geological concepts and knowledge

By starting from the log-constrained impedance inversion on a simple geological mode, the sources and behavior features of the ambiguity are tested and analyzed. Finally, a strategy for evaluating the reliability of inversion results is proposed.

\section{Fundamental principles}

Ignoring noise effects, seismic records can be expressed as

$$
s(t)=w(t) * \sum_{j=1}^{n-1} r_{j} \delta\left(t-T_{j}\right)
$$

where $w(\mathrm{t})$ is the seismic wavelet, $r_{\mathrm{j}}$ the reflection coefficient at time $T_{j}$, which can be expressed with impedance $z$ as

$$
r_{j}=\frac{z_{j}-z_{j-1}}{z_{j}+z_{j-1}}
$$

The partial derivative matrix A of Eq. (1) against impedance $z$ can be expressed as

$$
\begin{array}{r}
a_{i j}=\frac{\partial s(i \Delta t)}{\partial z_{j}}=\frac{2 z_{j-1}}{\left(z_{j}+z_{j-1}\right)} w(i \Delta t) * \delta\{[i-(j-1) \Delta t]\} \\
-\frac{2 z_{j+1}}{\left(z_{j}+z_{j+1}\right)} w(i \Delta t) * \delta[(i-j) \Delta t]
\end{array}
$$

Supposing the impedance vector after iterating $k-1$ times to be $Z_{k-1}$, and its corresponding synthetic seismic record vector to be $S_{k-1}$, the impedance vector $Z_{k}$ after iterating $k$ times is

$$
\begin{aligned}
\mathbf{Z}_{k}= & \mathbf{Z}_{k-1}+\mu \Delta \mathbf{Z}=\mathbf{Z}_{k-1} \\
& +\mu\left(\mathbf{A}_{k-1}^{T} \mathbf{A}_{k-1}+\lambda_{k} \mathbf{I}\right)^{-1} \mathbf{A}_{k-1}\left(\mathbf{S}-\mathbf{S}_{k-1}\right)
\end{aligned}
$$

where $\lambda_{k}$ is the damping factor, $\mu$ the relaxation factor, and $0<\lambda_{k}<1,0<\mu \leq 1$.

The basic flowchart of log-constrained inversion showed in Fig. 1 can be divided into the following steps.

1) The initial model is built by interpolating logging data along the geologic horizons;

2) The seismic records are synthesized with the initial model and wavelet by using a convolution method;

3 ) The error between synthetic records and real records is calculated;

4) The impedance model is modified and updated on the basis of back-propagating errors;

5) The final impedance model is obtained until the error between the synthetic and real seismic data is less than a given threshold value.

From the above procedure, it can be seen that logconstrained impedance inversion is a process in which the impedance model is iteratively modified so that the synthetic seismic data continuously approximate the real seismic data.

The constraint conditions of impedance inversion are geological information and logging data. Meanwhile, the convergence condition of impedance inversion is the minimum error between the synthetic and real seismic data (Li, 2008). Either the constraint condition or the convergence condition is used to evaluate the reliability of inversion results with the current method. This type of appraisal method is obviously deficient in persuasion.

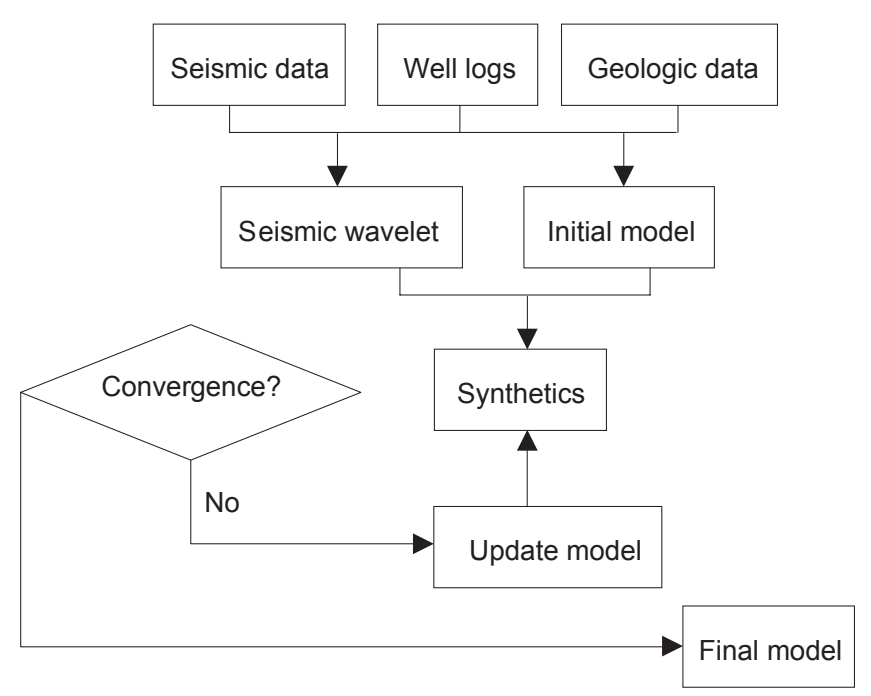

Fig. 1 Basic flowchart of log-constrained impedance inversion

\section{Inversion experiment on the model}

To analyze and evaluate the inversion method and the result it achieves, a geological model, as displayed in Fig. 2, is designed, on which 'blue' represents shale with a velocity of $2800 \mathrm{~m} / \mathrm{s}$ and a density of $2.29 \mathrm{~g} / \mathrm{cm}^{3}$; 'yellow' stands for sand with a velocity of $3000 \mathrm{~m} / \mathrm{s}$ and a density of $2.29 \mathrm{~g} / \mathrm{cm}^{3}$; 'red' represents igneous rock with a velocity of $3400 \mathrm{~m} / \mathrm{s}$ and a density of $2.36 \mathrm{~g} / \mathrm{cm}^{3}$. Considering that the algorithm of $\log$ constrained inversion is based on the convolution model, a 
convolution-based algorithm is also used in simulating the seismogram. Therefore, the inversion result can be free of the effects of multiples and converted-waves generated from the wave equation-based simulation algorithm. Fig. 3 is a synthetic seismogram using the convolution algorithm with a $26 \mathrm{~Hz}$ Ricker wavelet. To simulate the inversion process of real seismic data, four reflection events were tracked and interpreted, which correspond respectively to the top of the first horizontal sand layer, the bottom of the second horizontal sand layer, as well as the top and bottom of the wedge-shaped sand layer. Then six logs, named W1, W2, W3, W4, W5 and W6 in order, were created according to the geological model, with the odd ones going through the channel sand and igneous rock, but the even ones not. The four interpreted horizons and six logs are marked in the seismic section, as shown in Fig. 3.

Next, three experiments are done.

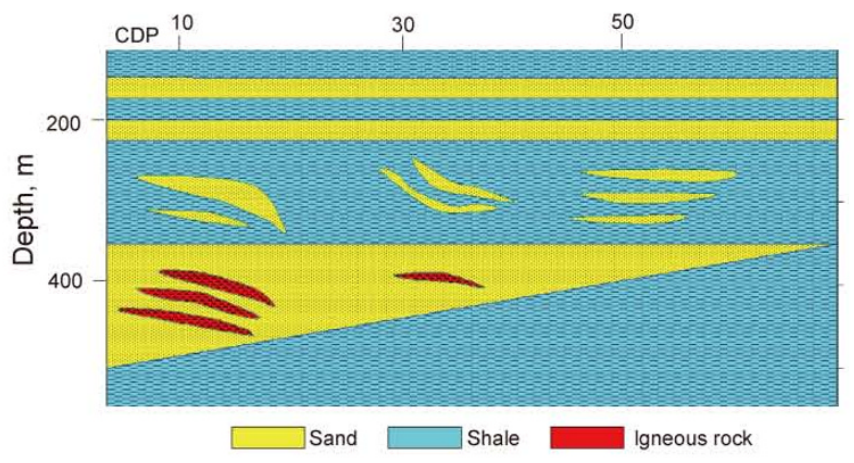

Fig. 2 Geological model

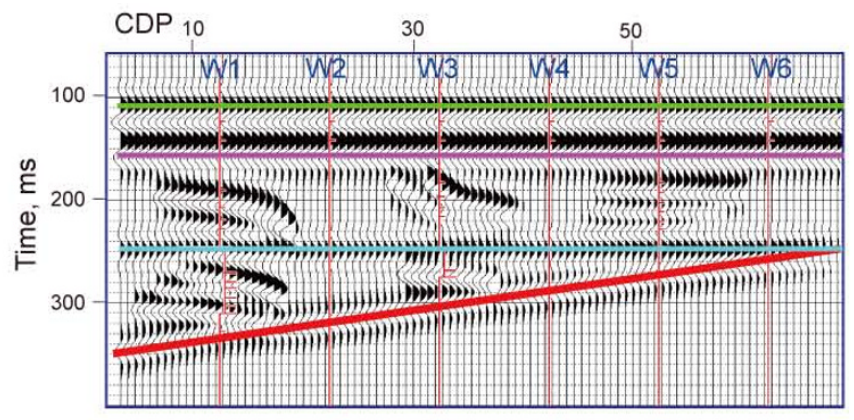

Fig. 3 Seismic data, interpreted horizons and well loggings corresponding to geological model

The first one is to analyze both the effects of the initial model on inversion results and the feasibility of the inversion method to establish the initial model. The initial impedance model is built by applying the interpolation of three evennumbered logs along the interpreted horizons. Note that the three wells do not go through the channel sand and igneous rock. Fig. 4(a) is the initial model on which there is no information of the channel sand and igneous rock. With the initial model and $26 \mathrm{~Hz}$ Ricker wavelet, the seismic data shown in Fig. 3 are inverted for impedance, and the result is shown in Fig. 4(b). Although the position and conformation of the channel sands and igneous rocks are found with converted results, compared with the geological model, the resolution of the channel sands between well W2 and W4 is far from expectation. The experiment indicates that on the one hand, the seismic data have a strong capability to modify and improve the initial model and on the other hand, the resolution of inversion results is limited when there are not enough high frequency components on the initial model.

The second experiment is to investigate the role of high frequency components of logging data during the inversion process and their effects on the inversion result. The second initial model is based on the interpolation of logging data along the interpreted horizons by using the three oddnumbered wells, and the three even-numbered wells are not used to build the second initial model, as shown in Fig. 5(a). Since the lateral variations of the sub-layers have not been suitably taken into account during the interpolation of logging data, the distribution of channel sands and igneous rocks in the second initial model deviated obviously from the geological model shown in Fig. 2. In practical inversion, only the major horizons, instead of all horizons, are tracked and interpreted. Otherwise, it is meaningless to invert the seismic data. Therefore, it is a normal phenomenon that the high frequency components on the initial model are different from those on the geological model. The key problem is whether the false high components of the initial model are updated and can converge to the correct solution after inversion. Fig. 5(b) is the inversion result. The resolution in Fig. 5(b) seems higher than that in Fig. 4(b), and more geological details seem to be found in Fig. 5(b). In fact, compared with the true geological model shown in Fig. 2, it can be seen that the footprint of the initial model can be easily found in the high frequency components in Fig. 5(b), and the apparent geological details in Fig. 5(b) are much of an artifact resulting from inversion.

The third experiment is to investigate whether the synthetic seismogram generated from the inverted impedance model can be used to evaluate the reliability of the inversion result. Fig. 6 is a synthetic seismogram corresponding to the inversion result. Yet, compared with the real seismic data shown in Fig. 2, the difference between them is hard to distinguish visually with a proportional error of less than $1 \%$. This experiment indicates that the consistency between the synthetic seismogram and real seismic data verifies the validity of the inversion algorithm. However, it cannot be used to evaluate the reliability of the inversion result.

\section{Appraisal of the inversion reliability}

From the above experiment it is seen that the reliability of the inversion result cannot be appraised objectively from the consistency of the synthetic seismogram with real seismic data. Another appraisal standard, usually used by geologists, is the consistency between the inverted trace around wells and logging data. In fact, since the seismic inversion is a process of starting from logging analyses and 


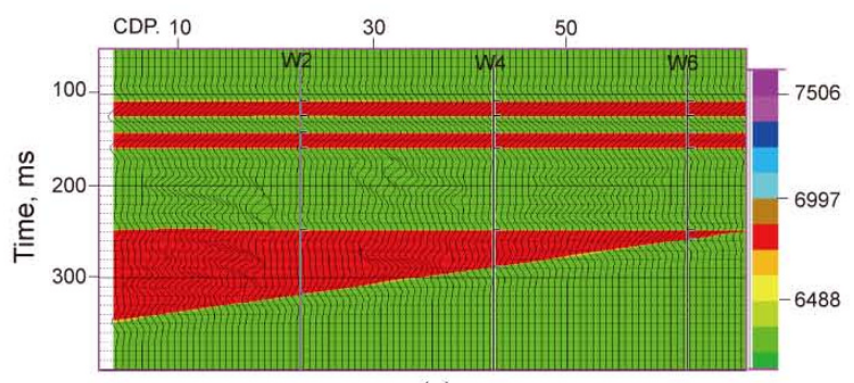

(a)

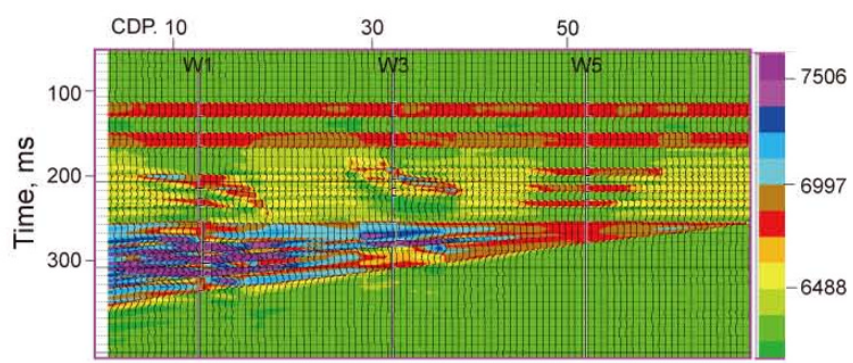

(b)

Fig. 4 (a) Initial model interpolated along horizons using well W2, W4 and W6 and (b) the inversion result

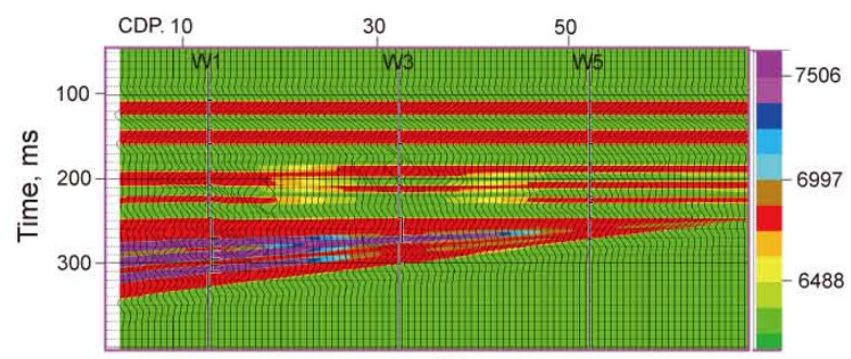

(a)

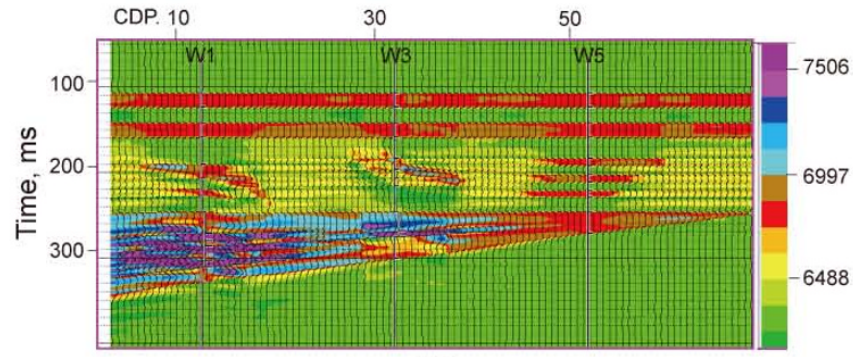

(b)

Fig. 5 (a) Initial model interpolated along horizons using well W1, W3 and W5 and (b) the inversion result

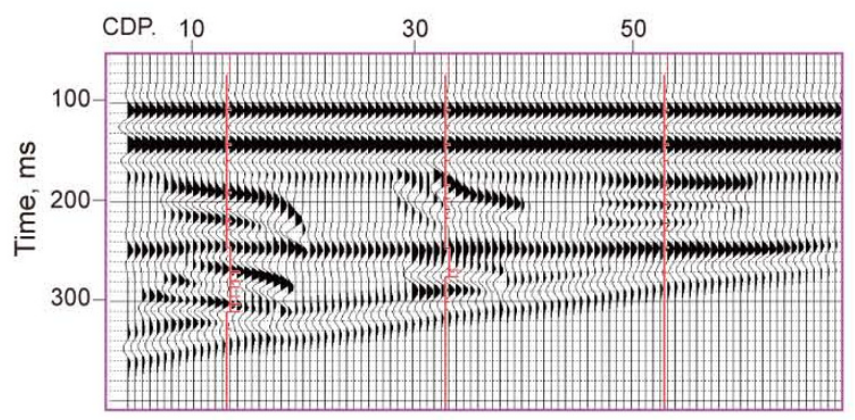

Fig. 6 Synthetic seismogram derived from the inversion result in Fig. 5(b) then returning to logging information, the iterative procedure itself ensures the inverted trace around wells to be less deviated from the logging data. Besides, even though they are quite consistent, the agreement at the well position cannot confirm the reliability of the inversion result between wells. One more appraisal method, sometimes used by geologists, is called 'blind well'. This is only part of the wells, used in an inversion process. The rest are used to verify the inversion result. Due to the intrinsic ambiguity of the inversion method and the constraint of logging data, the seemingly reasonable method reduces in fact the accuracy of the inversion result.

The impedance components within an effective frequency band, controlled by seismic data, cannot be ambiguous. The reliability of the inversion result depends mainly on the high frequency components beyond the effective frequency band. Therefore, the following strategy is proposed to evaluate the reliability of high frequency components. First, the inverted impedance model is transformed to a reflection coefficients section. Then the maximum effective frequency of real seismic data is chosen as a cut-off point, and the reflection coefficient section is decomposed into two components by low-pass and high-pass filtering. The lowfrequency component, within a seismic effective bandwidth, should have reflected characteristics similar to real seismic data. However, the high-frequency component, beyond the effective bandwidth, is much more ambiguous. Therefore, the emphasis of analysis and evaluation should be put on the high frequency component. If the geometrical reflection characteristics of the high frequency component are similar to the real seismic data, it is reliable. Otherwise, if its geometrical characteristics are far from real seismic data, its reliability is in doubt and should be verified.

Fig. 7(a) is a coefficient section transformed from the impedance model shown in Fig. 5(b). Compared with the original seismic data shown in Fig. 3, geometrical reflection characteristics of the horizontal sands and wedge sand bodies are similar to the real seismic data. However, the channel sand and igneous rock are much different from real seismic data. A further analysis should be made to verify the potential artifacts. Taking $80 \mathrm{~Hz}$ as a cutoff frequency point, the coefficient section shown in Fig. 7(a) is filtered respectively by low-pass and high-pass filters. Fig. 7(b) is the result from low-pass filtering, the Fig. 7(c) from high-pass filtering. The geometric reflection characteristics of the low frequency components shown in Fig. 7(b) are much closer to the real seismic data, which justifies low frequency components of inverted impedance as relatively reliable. In high frequency components shown in Fig. 7(c), the geometric reflection characteristics of channel sands and igneous rocks are far from real seismic data. For the igneous rock reflections in particular, their orientations, are visually opposed to the real seismic reflection, and the footprints of the initial model can be easily found from the igneous rock reflections as shown in Fig. 7(c). Therefore, it can be deduced that the high frequency components of inverted impedance shown in Fig. 5(b) are false, as they are artifacts resulting from the initial model. 


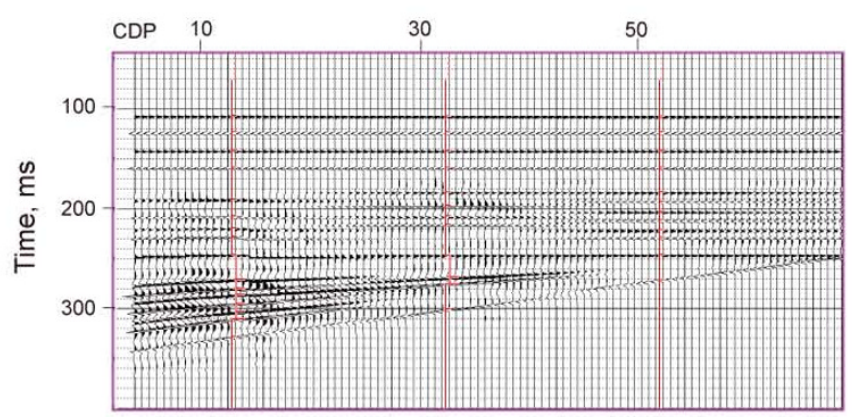

(a)

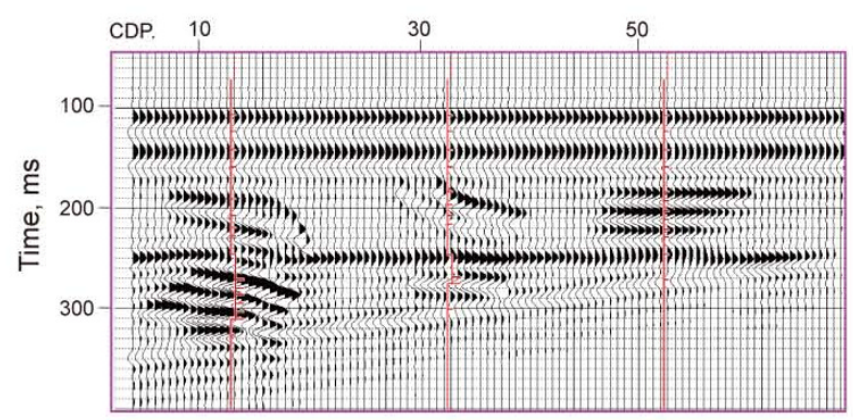

(b)

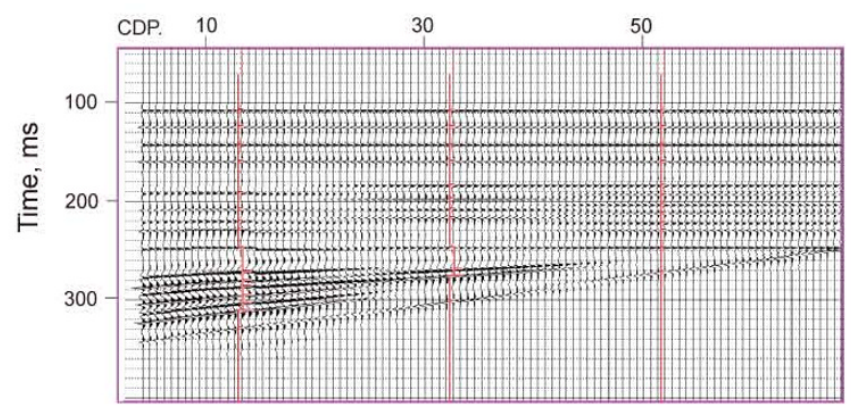

(c)

Fig. 7 (a) reflection coefficient section derived from the inversion result in Fig. 5(b), (b) its low frequency component and (c) high frequency component. The cutoff frequency is chosen as $80 \mathrm{~Hz}$.

\section{Conclusions}

Based on inversion experiments using a simple geological model, the following procedures are proposed on how to evaluate the reliability of the inversion result.

1) Transform the inverted impedance model to a reflection coefficient section;

2) Taking the maximum effective frequency of real seismic data as a cutoff frequency point, the reflection coefficient section is decomposed into two components by low-pass and high-pass filtering.

3) Taking the real seismic data as a reference, the geometric reflection characteristics of the high frequency components are investigated and analyzed. If the geometric characteristics of the high frequency components, such as the orientation and the distribution range, are similar to the real seismic data, the inversion results are reliable. Otherwise, the results are in doubt and should be further verified.
Although our experiments and analyses are based on logconstrained impedance inversion, the appraisal method is also practicable to sparse-pulse inversion, stochastic inversion and even elastic impedance inversion.

It should be noted that the importance of seismic inversion should not be neglected in reservoir prediction, while the ambiguity of inversion and the significance of the appraisal method are emphasized. On the contrary, seismic inversion technique will continue to play essential roles in reservoir prediction. In practical work, geophysicists should not exaggerate the accuracy and reliability but should objectively evaluate and use the inversion results.

\section{Acknowledgements}

This research was financially supported by the Major Basic Research Development Program of China's 973 Project (grant No. 2007CB209608) and the Science and Technology Innovation Foundation of CNPC (grant No. 2010D-50060301).

\section{References}

Cerney B. Uncertainties in low-frequency acoustic impedance models. The Leading Edge. 2007. 26(1): 74-86

Chen G J, Duan Z B, Ma Z T. Discussion of the popularity of impedance inversion; Petroleum Geophysics. 2003. 1(3): 7-10 (in Chinese)

Connolly P. Elastic impedance. The Leading Edge, 1999, 18(4):438-452

Cui Y C, Wu M, Li W X and Fu L Y. Nonlinear high-resolution impedance inversion method and its application. Geophysical Prospecting for Petroleum. 2007. 46(1): 1-12 (in Chinese)

Francis A. Acoustic impedance inversion pitfalls and some fuzzy analysis. The Leading Edge. 1997. 16(3): 275-283

Fu L Y. An artificial neural network theory and its application to seismic data processing. Ph. D. thesis. 1995. China University of Petroleum, Beijing (in Chinese)

Fu L Y. Joint inversion of seismic data about acoustic impedance. Geophysics. 2004. 69(4): 994-1004

Ghosh S K. Limitations on impedance inversion of band-limited reflection data. Geophysics. 2000. 65(3): 951-957

Huang H D, Zhang R W, Wei S P. Research on application of seismic nonlinear random inversion to reservoir prediction in the thin sandstone of continental deposits. Journal of Petroleum. 2009. 32(3): 386-390 (in Chinese).

Juve E. Uncertainties relating to impedance layering derived from poststack stratigraphic inversion. 1997, 67rd Annual International Meeting. SEG. Expanded Abstracts. 1606-1609

Latimer R B. An interpreter's guide to understanding and working with seismic-derived acoustic impedance data. The Leading Edge. 2000. 19(3): $242-256$

Li G F, Liao Q J, Wang S X, et al. Discussions of the horizon calibration based on well-log synthetic seismogram: Geophysical Prospecting for Petroleum. 2008. 47(2): 145-149 (in Chinese)

Li G F, Xiong J L, Zhou H. Seismic reflection characteristics of fluvial sand and shale interbedded layers. Applied Geophysics. 2008. (3): 219-229

Li Q Z. On strategy for seismic restricted inversion. Oil Geophysical Prospecting. 1998. 33(4): 423-438 (in Chinese)

Ma J F, Wang X J. Choice of the initial wave impedance value and the multi-solution of seismic trace inversion. Oil Geophysical Prospecting. 1999. 34(3): 332-340 (in Chinese)

Ma J F. Wang Xuejun, Jia Chunhuan et al, Study of constraint 
methodology for constrained impedance inversion. Geophysical Prospecting for Petroleum. 2000. 39(2): 52-63 (in Chinese)

Puryear C I, Castagna J P. Layer-thickness determination and stratigraphic interpretation using spectral inversion. Theory and application. Geophysics. 2008. 73(2): R37-R48

Qin Nan. Research on the reliability of log-constrained extrapolation inversion and well-ties coherence(in Chinese). Master Thesis. 2009. Institute of Geology and Geophysics. Chinese Academy of Science. Beijing

Velis D R. Stochastic sparse-spike deconvolution. Geophysics. 2008. 73(1): R1-R9

Wu M, Fu L Y and Li W X. A high-resolution nonlinear inversion method of reservoir parameters and its application to oil/gas exploration. Chinese Journal of Geophysics. 2008. 51(2): 546-557 (in Chinese)

Yang J H, Li G F, Liu Y. The application of elastic impedance inversion in reservoir prediction at the Jinan area of Tarim Oilfield. Applied Geophysics. 2007. 4(3): 201-206

Yao F C, Gan L D. Application and restriction of seismic inversion. Petroleum exploration and development. 2000. 27(2): 53-56 (in Chinese)

Zhang M Z, Yin X Y, Tao M Y, et al. Comprehension and application of log-constrained seismic impedance inversion. Oil Geophysical Prospecting. 2007. 42(6): 699-702 (in Chinese)

(Edited by Yang Lei) 